\title{
ERRATA
}

\section{On the computation of Mathieu functions}

by the Group "NUMERICAL ANALYSIS" at Delft University of Technology

(Journal of Engineering Mathematics, 7 (1973) 39-61)

In this paper some misprints occur, of which at least one is a serious error. A list of known misprints is given below:

Page 40 formula $4 \mathrm{c}$ should read:

$$
M_{S}^{(j)}(z, q)=\sum_{k=1}^{r}(-1)^{k+r} B_{2 k}^{(2 r)}\left\{J_{k-s}\left(u_{1}\right) Z_{k+s}^{(j)}\left(u_{2}\right)-J_{k+s}\left(u_{1}\right) Z_{k-s}^{(j)}\left(u_{2}\right)\right\} / B_{2 s}^{(2 r)}
$$

Page 48 line 3 should read:

$$
\mathrm{w}:=2 * \operatorname{order}+1 ; \mathrm{w} 2:=\mathrm{w} * \mathrm{w} ;
$$

Page 54 line 24 should read:

$$
\mathrm{Y}:=\mathrm{H} *(\mathrm{Q} * \mathrm{HC}+\mathrm{P} * \mathrm{HS}) ;
$$

Page 54 line 40 should read:

$$
\mathrm{A}[14]:=-0.0004606261662063 \text {; }
$$

Page 55 line 40 should read:

value $\mathrm{c}, \mathrm{x}$; integer $\mathrm{c}$; real $\mathrm{x}, \mathrm{j}, \mathrm{y}$; label alarm;

Page 55 line 44 should read:

begin real p, q, h, hc, hs; array a $[0: 30]$;

Page 56 lines 51 and 52 should read:

$$
\begin{aligned}
& \mathrm{h}:=0.7978845608028654 / \operatorname{sqrt}(\mathrm{x}) ; \\
& \mathrm{j}:=\mathrm{h} *(\mathrm{p} * \mathrm{hc}-\mathrm{q} * \mathrm{hs}) ;
\end{aligned}
$$

Page 58 lines 31-32 should read:

comment $5 * 10^{-11}$ is the relative accuracy of the i.c.l. 1905 computer;

for $\mathrm{m}:=\operatorname{mstar}-1$ step -1 until nmax do $\mathrm{p}:=1 /(\mathrm{m} * \mathrm{a}-\mathrm{p})$;

begin array $\mathrm{g}[\mathrm{k}+1: \mathrm{nmax}] ; \mathrm{g}[\mathrm{nmax}]:=\mathrm{p}$;

This error can be serious in the computation of Bessel functions.

Page 57 line 7 should read:

$$
\mathrm{a}[4]:=1.2879940988576776 \text {; }
$$


Page 59 lines 21-22 should read:

$\mathrm{j}, \mathrm{z}[-\operatorname{order} / 2+0.1: \mathrm{kmax}+\operatorname{order} / 2+1.9]: \mathrm{j}[\mathrm{k}]=\mathrm{J}_{\mathrm{k}}\left(\sqrt{ } / \mathrm{qe}^{-\mathrm{x}}\right)$

$\mathrm{z}[\mathrm{k}]=\mathrm{J}_{\mathrm{k}}\left(\sqrt{ } \mathrm{q} \mathrm{e}^{\mathrm{x}}\right)$ or $\mathrm{Y}_{\mathrm{k}}\left(\sqrt{ } \mathrm{q} \mathrm{e}^{\mathrm{x}}\right)$

Page 60 line 5 should read:

$$
\sin (k x)=\sin ((k-1) x) \cos (x)+\cos ((k-1) x) \sin (x)
$$

Page 60 line 11 should read:

$$
\operatorname{si}[\mathrm{k}]:=\mathrm{si}[\mathrm{k}-1] * \operatorname{co}[1]+\operatorname{co}[\mathrm{k}-1] * \mathrm{si}[1] \text {; end } \mathrm{k} \text {; }
$$

Page 60 line 24 should read:

if no $=4$ then begin ez: $=\exp (\mathrm{x}) ; \mathrm{u} 1:=\mathrm{h} / \mathrm{ez} ; \mathrm{u} 2:=\mathrm{h} * \mathrm{ez}$ end;

All these errors are due to incorrect copying of our original programs. Therefore our claim of accuracy in our results is not affected by them. 